\title{
1 DISCOURSES ON THE INTERACTION OF INFORMATION SYSTEMS, ORGANIZATIONS, AND SOCIETY: REFORMATION AND TRANSFORMATION
}

\author{
Richard Baskerville \\ Georgia State University \\ U.S.A. \\ Jan Stage \\ Aalborg University \\ Denmark
}

This book marks the turn of the first century of the information age. The purpose of the book is to provide ideas and results from past, current and future investigations of the relationships and interactions among four major components: information systems (IS), information technology (IT), organizations, and society. These investigations share a primary focus on the interrelationships, not on the components themselves.

The contributions to the book deal with history of information systems theory and technology, with the directions faced by those sharing the concerns of the field in its future research, and with attempts to draws these two views together. The authors provide essays and research reports as well as research proposals for future work in this area. They also provide well-substantiated integrative frameworks that facilitate recognition and transfer of relevant knowledge about the roles and uses of information technology. These frameworks are based on a wide range of disciplines such as philosophy, history, sociology, political science, management, and computer science.

We have organized the contributions to this book into five discourses. Collectively, the five discourses answer the key question: "What is the status of the discipline of information systems as we stand at the juncture of the new century?" Individually, the five discourses deal with the following issues: 
- Fundamentals: The basic concepts and models that are used for understanding and developing information systems.

- Challenges: The classical solutions and new problems that confront the field of information system.

- Conceptualization: The process of developing concepts and models that are used to understand and change information systems use and development.

- Automation: The interaction between humans and computers that is a key constituent part in an information system.

- Technology: The shape of the technology that is used to design and implement information systems.

In addition, we have aligned the contributions to each discourse with one of the following two perspectives:

- Reformation: The aim is to show how certain well-known issues are traditional and enduring, and that we can advance through revision and regeneration of issues from the past.

- Transformation: The aim is to show how other issues are entirely fresh and new, and that we can advance through generating issues that are completely unlike those of the past.

These two dimensions can be combined as illustrated in Figure 1. This table spans a framework for the contributions to this book. It should, however, be read with two limitations in mind. First, the vertical dimension does not represent a clear-cut division because the subjects are overlapping, and elements of the horizontal dimension are not always antithetical positions with an implied dialectic. Second, the positioning of a contribution in a cell of the table does not necessarily characterize the general perspective of any authors. Rather, the point is that the purpose, results, and conclusions of a particular contribution in this book align with the argument related to a specific discourse and perspective.

Based on this framework, the contributions are organized into nine parts. The first eight parts are defined by the first four discourses and each of their two perspectives. The ninth part concerns the new shapes in technology and its development (aligning generally with the transformational perspective). In the final part ten of the book, we include the descriptions of four panel discussions that regard research methods and the increasing distribution of organizations. 


\begin{tabular}{|l|l|l|}
\hline & \multicolumn{2}{|c|}{ Perspective } \\
\hline Discourse & Reformation & Transformation \\
\hline Fundamentals & Part 1 & Part 2 \\
& Jones & Davis \\
& Mumford & Braa and Rolland \\
& Boland & Russo \\
\hline Challenges & Part 3 & Part 4 \\
& Land & Markus \\
& Mathiassen & Kendall and Kendall \\
& Crowston & Walsham \\
\hline Conceptualization & Part 5 & Part 6 \\
& Sawyer & Kvasny and Truex \\
& Hirshheim and Klein & Introna and Ilharco \\
& Lamb and Davidson & Venable and Travis \\
\hline Automation & Part 7 & Part 8 \\
& Pouloudi and Whitley & Mark \\
& Aanestad and Hanseth & Grundén \\
& Rose and Truex & Karsten \\
\hline Technology & & Part 9 \\
& & Wilson and Howcroft \\
& & Eriksen \\
& & Berg \\
\hline
\end{tabular}

Figure 1. Discourses and Perspectives in this Book

\section{Reforming the Fundamentals}

The first discourse regards the reformation or transformation in the fundamentals of the information systems discipline. In this part, we ask "Are the most fundamental concepts in the field enduring, or are these fundamental concepts evolving?" The authors primarily adopt the perspective of reformation as they build on certain fundamental concepts that were relevant in the past and show how these are just as relevant today.

In "The Moving Finger: The Use of Social Theory in WG8.2 Conference Papers 1975-1999," Jones uses citation data from the corpus of IFIP WG 8.2 working conferences to uncover indications as to how the social theory that inhabits information systems research is evolving. WG 8.2 conferences give earlier and greater attention to social theorists than is typical for the IS field, and while this theoretical base appears to have emerged, significant and irreversible shifts are not apparent.

In "Socio-technical Design: An Unfulfilled Promise or a Future Opportunity," Mumford surveys the history and purposes of the socio-technical movement in information systems to reveal its future potential. The principles of socio-technical design, and particularly its value system, are even more strongly relevant to information systems in the coming "wired world" because new organizational forms and economic 
processes require careful management of the increasingly fluid mutual benefits between employers and employees.

Boland, in "The Limits of Language in Doing Systems Work," uses the imagery of a roundtable discussion between respected social philosophers to frame an inventory of current assumptions about language and systems. The fundamental roadblock to the realization or successful implementation of many good ideas for information technology is language. This limit is imposed because language bounds the ability of human beings to engage in systems thinking in myriad ways. The variety of these ways is revealed in a review of some of the fundamental philosophical assumptions underlying the systems literature.

\section{Transforming the Fundamentals}

In contrast, the authors in this section adopt a more revolutionary perspective regarding the fundamentals of the discipline. They tend to focus on the changes that are underway in the fundamental concepts in the field of information systems. These authors concentrate on how some of the most basic ideas in the future information systems arena will be essentially different from those of today.

In "Information Systems Conceptual Foundations: Looking Backward and Forward," Davis surveys past and potential formalisms and frameworks used to define our discipline. He finds that the concept of information systems as a discipline is aggregated from a set of technologies, methods, ideas, processes, etc. The members of this set are not individually persistent, although there are currently more arrivals than departures. The effect is to make the "core" concepts of information systems more difficult to distinguish because of varying assumptions, politics, and the current free-market of ideas.

Braa and Rolland, in "Horizontal Information Systems: Emergent Trends and Perspectives," use a case study to show how contemporary trends in information systems, such as internetworking, globalization, and enterprise resource planning, represent the emergence of horizontal, rather than vertical, information systems development. Institutional responses, such as standardization and knowledge management, create tension in the communities of practice because these conflict with the need for horizontal systems to be flexible, intra-active, and emergent.

Russo argues in "Expanding the Horizons of Information Systems Development" that electronic commerce is qualitatively changing the mix of skills and knowledge needed in systems development particularly regarding the need for technical skills in telecommunications and multi-media and business skills in collaboration and flexible management.

\section{Reforming the Classical Challenges}

While the authors above are focused on the essential ideas underlying the perspectives we take within the discipline of information systems, others look more closely at the essential solutions and problems we are choosing. Some of the work seems to suggest that the essential problems in information systems are enduring, and that we are overlooking 
the most fundamental and essential solutions to these enduring problems. These authors point to a reformation of some traditional problems, and address the question "Are we neglecting the most essential information systems solutions?"

In "Evaluation in a Socio-technical Context," Land argues that the failure of sociotechnical approaches to gain wider acceptance in IS practice may be largely due to the lack of acceptable ways of evaluating the organizational worth of the social elements within organizations. The discovery of practical techniques for accessing the social value of technology forms the main agenda for future socio-technical research.

Mathiassen uses the case of a large, long-term research project to construct a combined research approach to balance the diverse goals of research and practice. "Collaborative Practice Research" shows how professional work practices research is evolving into a hybrid of action research, experiments, and practice studies that enables the IS discipline to grow more closely attuned to the needs of practice.

Crowston argues in "Process as Theory in Information Systems Research" that the corpus of information systems research is ineffective because it is conceptually disconnected. Social-oriented research in information systems appears at multiple levels of analysis, e.g. at the individual level or at the organizational level. The recognized problem in linking research at these different levels of analysis can be resolved by centering the study of processes rather than the study of variance.

\section{Transforming Toward New Challenges}

On the other hand, other authors are suggesting that we are completely missing the right questions. These authors ask: “Are we choosing the right problems to solve?" These authors are suggesting that the essential problems have either been overlooked in the past or may have undergone revolutionary change over time. Therefore, they suggest that we are not concentrating our efforts on the real problems.

In "Toward an Integrated Theory of IT-related Risk Control," Markus argues that IS practices are growing inconsistent with recent management practice because IS has failed to achieve an integrated approach to risk management. An integrated view of IT-related risk includes system development failure, security breaches, and competitive threats, and enables intelligent, end-to-end tradeoff decisions in the management of IS.

The second chapter in this part is "Individual, Organizational, and Societal Perspectives on Information Delivery Systems: Bright and Dark Sides to Push and Pull Technologies" by Kendall and Kendall. They find that the benefits of push and pull information delivery systems may be outweighed by the social costs being unleashed on groups and individuals. The potential of each technology is described on four levels of increasing technological capabilities. The dangers include bandwidth wasted on unwanted content and the information overload, anxiety, addiction, and disorientation and the consequent stress, illness, and social dysfunction. There are remedies for these problems, ranging from unplugging to educating users and providers, but the nature and implications of the problem and the remedies are not yet understood.

Walsham in "Globalization and IT: Agenda for Research," finds us emerging into a century in which global information systems are ascendant, yet we are poorly prepared to use or understand these systems. We lack research that informs about the impact of 
these systems on human identity or on the effects of global groupware and intranets. We lack more critical organizational case studies and a sound multi-stakeholder perspective of the costs and benefits of global internetwork trading. We also lack a sense of the role and value of global IS in different cultures.

\section{Reformation of Conceptualizations}

Our authors also seem prepared to question whether our institutionalized "way of thinking" about information systems is the most useful way to approach the information systems issues facing us today. This process of conceptualization, this way of thinking, encompasses the processes that shape the way we apply fundamental concepts to finding the problems and resolving the solutions. These authors wonder whether the analytical approaches, mental models, and metaphors that inhabit our approach to the study of information systems are still right for the job. The authors in this part suggest that, in some ways, we should revise our institutionalized conceptualization processes. They are prepared to ask "Should we reform our old ways of thinking about these phenomena?"

In surveying the literature on multiple-method information systems research, Sawyer finds that the use of multiple research methodologies in each particular research study has not been well-explored in the information systems field. In "Studying Organizational Computing Infrastructures: Multi-method Approaches," Sawyer compares published examples with his own work and argues that multiple methodologies in the study of the application of any new information technology are in fact required by the need to study both the new technology and the organizational context. The technological infrastructure is too multi-faceted, i.e., unique, pervasive, context-driven, and emergent, for a monotonic study. Multi-method research suggests that multiple researchers, a priori theory, and an analytical dialectic should characterize information systems research.

In "Information Systems Research at the Crossroads: External Versus Internal Views," Hirschheim and Klein argue that non-IS practitioners have an unrealistic image of IS and its potential. IS academia is fragmented into non-interacting sects. The IS paradigms need to be adjusted to support better generalization and a broader view of both rigor and relevancy in order to establish the needed communication channels among IS academics, IS practitioners, and non-IS business professionals.

The final chapter in this part is "The New Computing Archipelago: Intranet Islands of Practice." By comparing the current development of organizational intranets with the past development of end user computing (EUC), Lamb and Davidson find intranet development is following the same pattern as EUC a generation earlier. Like EUC, intranets arise as islands of incompatible, independently developed technology without a shared vision or a common standard. The lure of centralized control over intranet development, standards and resources, at tension with the need to foster entrepreneurship and innovation, generates conflict over the role of IS professionals in relation to intranets, just as it did with EUC.

\section{Transformation of Conceptualizations}

In this part, we find authors whose work calls for entirely new conceptualization processes. These authors see the answer to this question, whether our institutionalized 
"way of thinking" about information systems is concordant with our needs, in a different light than the authors in part 5. They are prepared to ask instead, "Should we invent entirely new ways of thinking about information systems phenomena?"

The first chapter in this part is "Information Technology and the Cultural Reproduction of Social Order: A Research Paradigm." Using Bourdieu's theory of social practice as a framework, Kvasny and Truex show how information technology cannot seriously be used as a universal force for human empowerment because it has evolved as a cultural commodity that may only be acquired with cultural capital. Therefore, information technology can only be used to reproduce the existing social order in terms of enfranchising currently privileged actors while further disenfranchising the unprivileged.

In "The Screen and the World: A Phenomenological Investigation into Screens and Our Engagement in the World," Introna and Ilharco consider the significance of the growth to almost continuous engagement between people and their video screens. Video screens are so ubiquitous in current technology that we overlook the meaning that such screens assume for us. Screens "mediate our being in the world by presenting relevance in this world." Screens always present what matters, hide all else, and reveal a presumed a priori agreement about the viewer's situation in their world. Far from being simple, the concept of "screen-ness" is multi-faceted and loaded with meanings that information systems managers and designers ignore.

Venable and Travis, in "Developing a Virtual Community-based Information Systems Digital Library: A Proposal and Research Program," consider our contentment with the historically-fragmented repositories of human knowledge, calling upon us to reconsider the need for this distributed view of libraries. The information systems community should lead in developing an integrated, universal, information systems digital library. Proper development of this resource would involve a virtual community working as a group to develop a consensus regarding purpose and functionality. An amalgamation of soft systems and group methodologies may achieve this purpose.

\section{Reforming Automation}

The discourses move into a different plane as our authors in this part work to consider the most central facet in the interaction of information systems and organizations: automation of functions and the consequent human-machine interaction. It seems central to many of our authors that the relationships and exchanges between human beings and computing machinery are evolving. In particular, a continuous change is developing in the division of work between people and machines. In this part, we find work that reveals a deeper understanding of the interactions between people and machines, a revision and reformation of our previous thinking. The work of this group of authors collectively helps answer the question "Is the explosion of information technology actually changing the working boundaries between humans and machines?"

This part begins with "Representing Human and Non-human Stakeholders: On Speaking with Authority." Using an illustrative case study, Pouloudi and Whitley explore how an encryption algorithm became elevated to the level of other stakeholders in an information system setting. Non-human stakeholders, such as particular technologies, 
may become inscribed with human values. The observations of this study indicate how these values are not a singular cohesive set, but vary according to interpretation by the different human stakeholders.

In "Implementing Open Network Technologies in Complex Work Practices: A Case from Telemedicine," Aanestad and Hanseth use a case study to show how the introduction of broadband multimedia into organizations creates a hybrid collectif or collective of humans and non-humans. The interests of both technology and human actors must be translated for alignment through the dialog between an organization and its technology. Because this dialog is irreducibly interactional, it is impossible to plan or specify in advance, but must be cultivated in a process by which both the organization and the technology are socialized and educated as children in a new culture.

Rose and Truex, in "Machine Agency as Perceived Autonomy: An Action Perspective," consider machine agency, how technology seems to go about structuring human work. Contrasting theoretical positions, structuration and actor-network theories, can align when attributed machine autonomy is regarded. This attribution refers to the degree humans act according to a belief that the machine possesses autonomy. IT developers and managers need to consider this attribution more seriously because the closer one studies situated use, the more belief in autonomy is likely to be discovered. Consequently, agency theories can be seen themselves to be situated.

\section{Transforming Automation}

Another group of authors is suggesting revolutionary responses to this evolving relationship between human beings and computing machinery. These authors take a more normative stance, seeking new approaches to the design and construction of information systems that accommodate the changing boundaries. This work responds to the question, "Should we devise new ways to facilitate the interaction between humans and machines?"

In "Some Challenges Facing Virtually Colocated Teams," Mark is focussing on the challenges that arise when virtually colocated teams are to be supported by information technology. An empirical study and classical theories about interaction and group formation are used to analyze the differences between physically and virtually colocated teams, finding that technology shapes the culture of virtually colocated teams. It produces immediate and long-term behavioral effects on team development and effectiveness in terms of trust, motivation, cooperation, and patterns in participation and communication.

Grundén, in "MOA-S: A Scenario Model For Integrating Work Organization Aspects into the Design Process of CSCW Systems," concludes that work in CSCW has not developed good design methods to deal with the flexible character of either the technology or the organization, nor studied the consequences of CSCW for work processes. Using a case study approach, Grundén shows how the process of designing CSCW systems is improved by a model based on scenarios for articulating and discussing future designs of the work organization.

The final chapter in this part is "Constructing Interdependencies with Collaborative Information Technology." Karsten combines a theoretical approach with reviews of earlier empirical cases to discover that the relationships between information technology 
and social interdependence can be usefully interpreted by understanding four essentials. First, interdependence can originate through IT-mediated social integration. Second, time and space distanciation is enabled by stored information accessible through telecommunication. Third, the IT may situate or historically frame the institutionalization of social interdependence. Fourth, reciprocity in the storage of information can ingrain systemness, thereby transforming social integration to system integration.

\section{Transforming into New Shapes of Technology}

The last discourse we discover in the work of our authors regards important new ways to meet the new century of information systems development. These authors consider how the evolving worlds of technologies and social interaction inspire us to invent revolutionary new ways to shape the problems and solutions involved developing new information systems.

Wilson and Howcroft, in "The Role of Gender in User Resistance and Information Systems Failure," challenge the technical-economic view of failure and show how it is also a social construction. Using a case study of a hospital information system, they show how the role of gender in information systems failure is important because feminine values, such as hands-on caring, may be suppressed by the increase in abstract processing inherent in information technology. Discovery of gender issues may show that care can prevail and society and organizations can indeed benefit from a "technical" information system failure.

The next chapter is "Limitations and Opportunities of System Development Methods in Web Information System Design." Using a research approach that is interventiondriven and practice-oriented, Eriksen studies whether current systems development methods are appropriate for web design. He shows how newer information technologies, such as web technology, are changing social relationships by creating an "audience" for organizations. Design work in this shifting social context requires a distinction between producer and consumer communities and between interior and exterior design. Further, design methods must be concerned with dividing operational responsibility and maintaining the site information. Hypermedia methodology is shown to be useful to determine input specifications instead of formally described work tasks.

The final chapter is, perhaps appropriately, "Lessons from a Dinosaur: Mediating IS Research Through an Analysis of the Medical Record." Berg takes up the classical technical approach that still underlies information system development and thereby influences the way we shape the interaction between human beings and computers. The case of a paper-based versus electronic patient record is used to illustrate the inadequacy of the technical approach and the need for an interpretive approach in developing a satisfactory electronic solution.

\section{Panels on Research Methods and Distributed Organizations}

Two additional issues of a very basic nature inhabit the chapters in the first nine parts of the book. These issues are research methodology and new organizational forms. The first issue regards the problems involved in studying the complex social context of 
technology in human work. The second issue regards both the new ways in which humans can interact using technology and the ways this technology limits human action. In addition to the research papers presented in the other parts, this section includes brief descriptions of four panel discussions that deal exclusively with these two themes.

\section{Conclusion: Directions of the Collective Work}

Collectively, the work in the ten parts of this book comprises a discourse on a new horizon for our understanding of the interaction between human beings in organizations and society and their information systems. There are essays, theoretical papers, and empirical research reports about the changing roles and new impacts of information technologies in specific organizational contexts. These contributions suggest the new ways and means by which organizations can improve the design, implementation, and maintenance of information technologies. These contributions reveal the changing role that information technology plays in the lives of people as individuals and as members of complex social institutions such as government, community, business, professional societies, and other forms of social associations.

These changes are complex and dramatic. Much worth is discovered in our traditional thinking about information systems. The contributions to this book show exactly how to regenerate some well-known thinking in terms of fundamental concepts, solutions to known problems, conceptualization processes, and the interaction between people and machines. Worth is also discovered in revolutionary thinking for some issues. We call for completely new thinking in terms in other fundamental concepts, totally new problems, new conceptualization processes, new boundaries between people and machines, and new ways of shaping technologies.

What is our story as we turn the first century of the information age? We believe that certain important fundamental concepts remain relevant. Significant and irreversible shifts in social theory have not appeared, information technology is still central in the social interaction in organizations, and indeed has become more critical because the traditional mutual benefits between employers and employees has become more fluid. As always, human language barriers remain the fundamental roadblocks to IT implementation. We believe other transformational concepts are arising, and indeed our set of concepts is growing because there are more useful ideas arriving than are departing. For example, we need to distinguish the newer horizontal, rather than vertical, forms of information systems development and recognize the changing mix of skills and knowledge required in this area.

Many traditional problems remain to be solved, like finding acceptable ways of evaluating the organizational worth of social elements, developing better research methods, and linking our discoveries into a system. Possible solutions include developing hybrid methods that link research and practice, or centering processes for integrating theories. We are also becoming aware of new or unrecognized problems, such as the lack of any integrated approaches to risk management, the social costs being unleashed on people by the wiring of society, and our headlong rush into globalized systems without a proper understanding. 
We believe our conceptualization processes are inadequate. Some need revision, such as accepting that multiple methodologies are not just desirable, but necessary for information systems research. We need to adjust our paradigms to establish better communication channels throughout the field. We have to realize that the old end user tension between central control and innovation has reappeared in the intranet world. Innovative conceptualization processes are also needed. The video screen is becoming the central means for discovering our relevance to our universe, and now we must be prepared to approach information technology as a cultural commodity that may only be acquired with cultural capital. We are at the threshold of integrated, universal digital libraries where access to all of humanity's culture, our art, history, and technology, is neatly rolled and accessed in a virtual Library of Alexandria.

There has been an incredible evolution in the relationships and exchanges between human beings and computing machinery. Machines are acquiring human status, not through robotics, but rather through reification. Computers have become inscribed socially with autonomy and human values, and organizations have become hybrid collectives of humans and non-humans. Consequently we need new norms for defining the relationships and exchanges between human beings and computers. We need methods that permit designers to appreciate how technology shapes the culture of virtually colocated people, deals with flexibility that is absolutely necessary in both information technology and organizations, and allows designers to interpret information technology in relation to the social interdependence of organizational members.

Information technology has not only broken through technical barriers, but through social ones as well. We must raise our awareness of the social issues to the same new plane as that reached by technology. For example, we can see clearly the role of gender in defining information systems success or failure. We can see how web design implies changing an incredible web of social relationships. We can also see how rationaltechnical information systems design has endured even more unreasonably than rationaltechnical information systems management, and that the need for interpretive approaches for systems development is paramount.

We believe the new century raises on our horizon other complicated issues that we were unable to deal with in this volume. One of these issues is the ethics of these new IS and IT concepts and practices. The changes suggested above reveal to us many raw ethical dilemmas which arise in the development, use, and consequences of information technology, or in research about such technology. Many questions about the new century of information systems are answered in the work presented in this volume. However, we recognize that the ethical problems deserve more future space in our thinking and our research than was afforded here, and levy this as a challenge to future authors in this series.

\section{About the Authors}

Richard Baskerville is an associate professor in the Department of Computer Information Systems at Georgia State University. His research focuses on security and methods in information systems, their interaction with organizations and research methods. He is an associate editor of The Information Systems Journal and MIS Quarterly. Richard's 
practical and consulting experience includes advanced information system designs for the U.S. Defense and Energy Departments. He is a former chair of the IFIP Working Group 8.2, and a Chartered Engineer under the British Engineering Council. Richard holds MSc and Ph.D. degrees from the London School of Economics. He can be reached by e-mail at baskerville@acm.org.

Jan Stage is an associate professor in Computer Science at Aalborg University. His research interests include methods for analysis and design in software engineering, objectoriented analysis and design, and analysis and design patterns. His articles have appeared in MIS Quarterly, Information and Software Technology, Information Technology and People, and The Scandinavian Journal of Information Systems. He is a co-author of Object-Oriented Analysis and Design (in Danish and Swedish) and holds a Ph.D. in Computer Science from Oslo University. Jan can be reached by e-mail at jans@, intermedia.auc.dk. 